\section{Is human nature hiddine in the genome?}

\author{
Human Natures: Genes, Cultures and the Human Prospect \\ By Paul R. Ehrlich \\ Island Press, \$29.95, ISBN 1-55963-779-X, 2000
}

\section{Reviewed by John Tooby}

Center for Evolutionary Psychology and Department of Anthropology, University of California, Santa Barbara, California, USA

Common sense, some say, is what tells us the world is flat. Certainly sciences from quantum mechanics to cosmology prospered as we freed ourselves from the self-evident but erroneous concepts we reasoned with. Unfortunately, Paul Ehrlich is not someone who scrutinizes the concepts that lead him to his conclusions, and so Human Natures suffers from his trademark confidence in poorly chosen assumptions.

Ehrlich rose to fame by unpacking the logic inherent in the concept of resources (that is, they are physical, zero-sum, scarce, unexpandable) to argue that saving the lives of hundreds of millions of the world's desperate and hungry was a waste of resources that ought to be stopped. Surpluses, in his view, needed to be hoarded for the minority that could come through the impending resource crunch intact. Dissect the concept of resources, however, and you find that-despite common sense-they are partly made from information and so have been expanding exponentially for centuries. Advances in knowledge transform the worthless-grass seed, ore, petroleum, mold secretions, electromagnetic bandwidth, silicon, the DNA that adds beta-carotene to rice-into large, nonlinear expansions in production. In defiance of Ehrlich's recommendations, resource creation and aid have delivered millions from starvation, and show every sign of bringing humanity in for a demographic soft-landing, all without the need for the well fed to condemn humanity's poorest two-thirds to extinction.

Population remains a theme in Human Natures, but its major focus is evolutionary psychology, which Ehrlich mischaracterizes as a hereditarian theory that 'genes control' far more 'behaviors' than they actually do or could. His common sense tells him that 'genetic control' and 'learning' are two distinct and opposed ways of causing behavior, and if something is learned, genes must ipso facto be irrelevant. Re-inventing the traditional social science rationale for dismissing biology, Ehrlich reasons that because the "hallmark of our natures is incredible plasticity," and "genetic control" is "inflexible," genes must be unimportant for understanding human behavior. His broadside, however, never gets within shooting distance of its target because Ehrlich is unacquainted with the basic distinctions or hard evidence that have transformed this area of science.

To begin with, evolutionary psychology originated not as an endorsement of the idea that genes directly 'control' behavior (as Ehrlich has it), but famously as a rejection of that position. Indeed, evolutionary psychologists, many of whom have training both in evolutionary biology and cognitive science, not only accept but champion Ehrlich's favorite tenet that learning (and more broadly, input-driven neural computation) underlies almost all human behavior. But what for Ehrlich is the end of the story-our brains are largely learning systems - is for evolutionary psychologists, the beginning. Rather than accepting learning as an omnipotent, magical black box, they insist on breaking open the box to map the circuit logic through which its programs solve adaptive problems.

In one of those surprising scientific twists that turns received wisdom and common sense inside out, researchers have found that our learning programs are anything but blank slates. Blank-slate-like models have lost credibility because they were found to be computationally too feeble to solve many-and perhaps all-of the diverse array of adaptive problems that humans and other species routinely solve. To be powerful enough to accomplish their tasks, our neurocomputational programs must be numerous, diverse, specialized for particular functions, and come equipped by evolution with a battery of design features tailored to exploit the typical features of their respective problems. The surprise is that evolutionary and learning accounts, after their deficiences are corrected, turn out to be identical — rather than opposedtheories. Learning systems must be densely interpenetrated with genetically specified internal structure in order to function intelligently. Analogously, programmers know that the power of a programming environment is enhanced rather than made inflexible' by each addition to its library of subroutines, and computer users know that with each additional plug-in, their browser can read more formats, access more data, and perform more interesting tricks. Humans are flexible and intelligent precisely because we have evolved subroutines for vision, cooperation, language, friendship, mating, foraging, navigation, incest avoidance, aggression, and scores of other functions.

Because Ehrlich is unaware that gene action often works within the structure of learning, he consistently reports findings that contradict his view as if they supported it. For example, he cites experiments showing that macaques develop fear of snakes when they see others express fear toward snakes as a falsification of the idea that snake phobias were shaped by selection (after all, it's 'learned'). But he misses the significance of the central experimental finding: macaques who see others express fear toward non-snake stimuli do not come to fear these stimuli. That is, the particular learning system responsible was specialized by natural selection for regulating fear of snakes; the learning outcome is derived as much from the evolved organization of the circuitry as from the input.

The one genuinely interesting argument Ehrlich marshals is that the human genome is too small to encode much more than a blank slate. For Ehrlich, a gene is self-evidently something that encodes a protein, and by this definition our genes number only 30,000. For Darwinians, however, a gene is any nucleotide sequence whose modification would lead to a different developmental outcome. By this definition, the number of genes in our genome is presently unknown but orders of magnitude higher. The false assumption here is that it takes more types of building material (proteins) to build a larger or more complex structure. Instead, it only takes a more complex developmental plan. Knowledge of how the genome regulates development is only in its infancy, but already there is evidence that even the vast regions of noncoding DNA participate in the complex, dynamic system of feedback and control of gene expression. The human genome is roomy enough for a richly sculpted neural architecture, a universal human nature. Despite Ehrlich's well-meaning arguments, the world may turn out to be round, and the future territory of genetics may include not only learning mechanisms, but the behavior that learning makes possible. 